Kragujevac Journal of Mathematics

Volume 44(3) (2020), Pages 369-378.

\title{
ON THE HERMITE-HADAMARD TYPE INEQUALITIES FOR FRACTIONAL INTEGRAL OPERATOR
}

\author{
H. YALDIZ ${ }^{1}$ AND M. Z. SARIKAYA ${ }^{2}$
}

\begin{abstract}
In this paper, using a general class of fractional integral operators, we establish new fractional integral inequalities of Hermite-Hadamard type. The main results are used to derive Hermite-Hadamard type inequalities involving the familiar Riemann-Liouville fractional integral operators.
\end{abstract}

\section{INTRODUCTION}

Let $f: I \subseteq \mathbb{R} \rightarrow \mathbb{R}$ be a convex mapping defined on the interval $I$ of real numbers and $a, b \in I$, with $a<b$. The following double inequality is well known in the literature as the Hermite-Hadamard inequality [5]:

$$
f\left(\frac{a+b}{2}\right) \leq \frac{1}{b-a} \int_{a}^{b} f(x) d x \leq \frac{f(a)+f(b)}{2} .
$$

The most well-known inequalities related to the integral mean of a convex function are the Hermite-Hadamard inequalities.

In [2], Dragomir and Agarwal proved the following results connected with the right part of (1.1).

Lemma 1.1. Let $f: I^{\circ} \subseteq \mathbb{R} \rightarrow \mathbb{R}$ be a differentiable mapping on $I^{\circ}, a, b \in I^{\circ}$ with $a<b$. If $f^{\prime} \in L[a, b]$, then the following equality holds:

$$
\frac{f(a)+f(b)}{2}-\frac{1}{b-a} \int_{a}^{b} f(x) d x=\frac{b-a}{2} \int_{0}^{1}(1-2 t) f^{\prime}(t a+(1-t) b) d t .
$$
ity.

Key words and phrases. Fractional integral operator, convex function, Hermite-Hadamard inequal-

2010 Mathematics Subject Classification. Primary: 26A33. Secondary: 26D10, 26D15, 33E20.

DOI 10.46793/KgJMat2003.369Y

Received: September 24, 2017.

Accepted: May 22, 2018. 
Theorem 1.1. Let $f: I^{\circ} \subseteq \mathbb{R} \rightarrow \mathbb{R}$ be a differentiable mapping on $I^{\circ}, a, b \in I^{\circ}$ with $a<b$. If $\left|f^{\prime}\right|$ is convex on $[a, b]$, then the following inequality holds:

$$
\left|\frac{f(a)+f(b)}{2}-\frac{1}{b-a} \int_{a}^{b} f(x) d x\right| \leq \frac{(b-a)}{8}\left(\left|f^{\prime}(a)\right|+\left|f^{\prime}(b)\right|\right) .
$$

Meanwhile, in [8], Sarikaya et al. gave the following interesting Riemann-Liouville integral inequalities of Hermite-Hadamard type.

Theorem 1.2. Let $f:[a, b] \rightarrow \mathbb{R}$ be a positive function with $0 \leq a<b$ and $f \in L_{1}([a, b])$. If $f$ is a convex function on $[a, b]$, then the following inequalities for fractional integrals hold:

$$
f\left(\frac{a+b}{2}\right) \leq \frac{\Gamma(\alpha+1)}{2(b-a)^{\alpha}}\left[J_{a+}^{\alpha} f(b)+J_{b-}^{\alpha} f(a)\right] \leq \frac{f(a)+f(b)}{2}
$$

with $\alpha>0$.

Lemma 1.2. Let $f:[a, b] \rightarrow \mathbb{R}$ be a differentiable mapping on $(a, b)$ with $a<b$. If $f^{\prime} \in L[a, b]$, then the following equality for fractional integrals holds:

$$
\begin{aligned}
& \frac{f(a)+f(b)}{2}-\frac{\Gamma(\alpha+1)}{2(b-a)^{\alpha}}\left[J_{a+}^{\alpha} f(b)+J_{b-}^{\alpha} f(a)\right] \\
= & \frac{b-a}{2} \int_{0}^{1}\left[(1-t)^{\alpha}-t^{\alpha}\right] f^{\prime}(t a+(1-t) b) d t .
\end{aligned}
$$

Theorem 1.3. Let $f:[a, b] \rightarrow \mathbb{R}$ be a differentiable mapping on $(a, b)$ with $a<b$. If $\left|f^{\prime}\right|$ is convex on $[a, b]$, then the following inequality for fractional integrals holds:

$$
\begin{aligned}
& \left|\frac{f(a)+f(b)}{2}-\frac{\Gamma(\alpha+1)}{2(b-a)^{\alpha}}\left[J_{a+}^{\alpha} f(b)+J_{b-}^{\alpha} f(a)\right]\right| \\
\leq & \frac{b-a}{2(\alpha+1)}\left(1-\frac{1}{2^{\alpha}}\right)\left[\left|f^{\prime}(a)\right|+\left|f^{\prime}(b)\right|\right] .
\end{aligned}
$$

For some recent results connected with fractional integral inequalities see ([8]-[11]) In [7], Raina defined the following results connected with the general class of fractional integral operators

$$
\mathcal{F}_{\rho, \lambda}^{\sigma}(x)=\mathcal{F}_{\rho, \lambda}^{\sigma(0), \sigma(1), \ldots}(x)=\sum_{k=0}^{\infty} \frac{\sigma(k)}{\Gamma(\rho k+\lambda)} x^{k}, \quad \rho, \lambda>0,|x|<\mathcal{R},
$$

where the coefficents $\sigma(k), k \in \mathbb{N}_{0}=\mathbb{N} \cup\{0\}$, is a bounded sequence of positive real numbers and $\mathcal{R}$ is the real number. With the help of (1.7), Raina and Agarwal et al. defined the following left-sided and right-sided fractional integral operators, respectively, as follows:

$$
\begin{aligned}
& \mathcal{g}_{\rho, \lambda, a+; \omega}^{\sigma} \varphi(x)=\int_{a}^{x}(x-t)^{\lambda-1} \mathcal{F}_{\rho, \lambda}^{\sigma}\left[\omega(x-t)^{\rho}\right] \varphi(t) d t, \quad x>a, \\
& \mathcal{\partial}_{\rho, \lambda, b-; \omega}^{\sigma} \varphi(x)=\int_{x}^{b}(t-x)^{\lambda-1} \mathcal{F}_{\rho, \lambda}^{\sigma}\left[\omega(t-x)^{\rho}\right] \varphi(t) d t, \quad x<b,
\end{aligned}
$$


where $\lambda, \rho>0, \omega \in \mathbb{R}$, and $\varphi(t)$ is such that the integrals on the right side exists.

It is easy to verify that $\mathcal{J}_{\rho, \lambda, a+; \omega}^{\sigma} \varphi(x)$ and $\mathcal{J}_{\rho, \lambda, b-; \omega}^{\sigma} \varphi(x)$ are bounded integral operators on $L(a, b)$, if

$$
\mathfrak{M}:=\mathcal{F}_{\rho, \lambda+1}^{\sigma}\left[\omega(b-a)^{\rho}\right]<\infty .
$$

In fact, for $\varphi \in L(a, b)$, we have

$$
\left\|\partial_{\rho, \lambda, a+; \omega}^{\sigma} \varphi(x)\right\|_{1} \leq \mathfrak{M}(b-a)^{\lambda}\|\varphi\|_{1}
$$

and

$$
\left\|\mathcal{\partial}_{\rho, \lambda, b^{-} ; \omega}^{\sigma} \varphi(x)\right\|_{1} \leq \mathfrak{M}(b-a)^{\lambda}\|\varphi\|_{1}
$$

where

$$
\|\varphi\|_{p}:=\left(\int_{a}^{b}|\varphi(t)|^{p} d t\right)^{\frac{1}{p}} .
$$

The importance of these operators stems indeed from their generality. Many useful fractional integral operators can be obtained by specializing the coefficient $\sigma(k)$. Here, we just point out that the classical Riemann-Liouville fractional integrals $I_{a^{+}}^{\alpha}$ and $I_{b^{-}}^{\alpha}$ of order $\alpha$ defined by (see, $[3,4,6])$

$$
\left(I_{a^{+}}^{\alpha} \varphi\right)(x):=\frac{1}{\Gamma(\alpha)} \int_{a}^{x}(x-t)^{\alpha-1} \varphi(t) d t, \quad x>a, \alpha>0
$$

and

$$
\left(I_{b^{-}}^{\alpha} \varphi\right)(x):=\frac{1}{\Gamma(\alpha)} \int_{x}^{b}(t-x)^{\alpha-1} \varphi(t) d t, \quad x<b, \alpha>0,
$$

follow easily by setting

$$
\lambda=\alpha, \sigma(0)=1 \text { and } w=0
$$

in (1.8) and (1.9), and the boundedness of (1.13) and (1.14) on $L(a, b)$ is also inherited from (1.11) and (1.12), (see [1]).

In this paper, using a general class of fractional integral operators, we establish new fractional integral inequalities of Hermite-Hadamard type. The main results are used to derive Hermite-Hadamard type inequalities involving the familiar RiemannLiouville fractional integral operators.

\section{MAin Results}

In this section, using fractional integral operators, we start with stating and proving the fractional integral counterparts of Lemma 1.1, Theorem 1.1 and Theorem 1.2. Then some other refinements will folllow. We begin by the following theorem. 
Theorem 2.1. Let $\varphi:[a, b] \rightarrow \mathbb{R}$ be a convex function on $[a, b]$, with $a<b$, then the following inequalities for fractional integral operators hold:

$$
\begin{aligned}
\varphi\left(\frac{a+b}{2}\right) & \leq \frac{1}{2(b-a)^{\lambda} \mathcal{F}_{\rho, \lambda+1}^{\sigma}\left[\omega(b-a)^{\rho}\right]}\left[\left(\partial_{\rho, \lambda, a+; \omega}^{\sigma} \varphi\right)(b)+\left(\partial_{\rho, \lambda, b^{-} ; \omega}^{\sigma} \varphi\right)(a)\right] \\
& \leq \frac{\varphi(a)+\varphi(b)}{2}
\end{aligned}
$$

with $\lambda>0$.

Proof. For $t \in[0,1]$, let $x=t a+(1-t) b, y=(1-t) a+t b$. The convexity of $\varphi$ yields

$$
\varphi\left(\frac{a+b}{2}\right)=\varphi\left(\frac{x+y}{2}\right) \leq \frac{\varphi(x)+\varphi(y)}{2},
$$

i.e.,

$$
2 \varphi\left(\frac{a+b}{2}\right) \leq \varphi(t a+(1-t) b)+\varphi((1-t) a+t b) .
$$

Multiplying both sides of $(2.3)$ by $t^{\lambda-1} \mathcal{F}_{\rho, \lambda}^{\sigma}\left[\omega(b-a)^{\rho} t^{\rho}\right]$, then integrating the resulting inequality with respect to $t$ over $[0,1]$, we obtain

$$
\begin{aligned}
& 2 \varphi\left(\frac{a+b}{2}\right) \int_{0}^{1} t^{\lambda-1} \mathcal{F}_{\rho, \lambda}^{\sigma}\left[\omega(b-a)^{\rho} t^{\rho}\right] d t \\
\leq & \int_{0}^{1} t^{\lambda-1} \mathcal{F}_{\rho, \lambda}^{\sigma}\left[\omega(b-a)^{\rho} t^{\rho}\right] \varphi(t a+(1-t) b) d t \\
& +\int_{0}^{1} t^{\lambda-1} \mathcal{F}_{\rho, \lambda}^{\sigma}\left[\omega(b-a)^{\rho} t^{\rho}\right] \varphi((1-t) a+t b) d t .
\end{aligned}
$$

Calculating the following integrals by using (1.7), we have

$$
\begin{aligned}
& \int_{0}^{1} t^{\lambda-1} \mathcal{F}_{\rho, \lambda}^{\sigma}\left[\omega(b-a)^{\rho} t^{\rho}\right] d t=\mathcal{F}_{\rho, \lambda+1}^{\sigma}\left[\omega(b-a)^{\rho}\right], \\
& \int_{0}^{1} t^{\lambda-1} \mathcal{F}_{\rho, \lambda}^{\sigma}\left[\omega(b-a)^{\rho} t^{\rho}\right] \varphi(t a+(1-t) b) d t \\
= & \frac{1}{(b-a)^{\lambda}} \int_{a}^{b}(b-x)^{\lambda-1} \mathcal{F}_{\rho, \lambda}^{\sigma}\left[\omega(b-x)^{\rho}\right] \varphi(x) d x
\end{aligned}
$$

and

$$
\int_{0}^{1} t^{\lambda-1} \mathcal{F}_{\rho, \lambda}^{\sigma}\left[\omega(b-a)^{\rho} t^{\rho}\right] \varphi((1-t) a+t b) d t
$$




$$
=\frac{1}{(b-a)^{\lambda}} \int_{a}^{b}(x-a)^{\lambda-1} \mathcal{F}_{\rho, \lambda}^{\sigma}\left[\omega(x-a)^{\rho}\right] \varphi(x) d x .
$$

As consequence, we obtain

$$
2 \mathcal{F}_{\rho, \lambda+1}^{\sigma}\left[\omega(b-a)^{\rho}\right] \varphi\left(\frac{a+b}{2}\right) \leq \frac{1}{(b-a)^{\lambda}}\left[\left(\partial_{\rho, \lambda, a+; \omega}^{\sigma} \varphi\right)(b)+\left(\partial_{\rho, \lambda, b^{-} ; \omega}^{\sigma} \varphi\right)(a)\right]
$$

and the first inequality is proved.

Now, we prove the other inequality in $(2.1)$, Since $\varphi$ is convex, for every $t \in[0,1]$, we have

$$
\varphi(t a+(1-t) b)+\varphi((1-t) a+t b) \leq \varphi(a)+\varphi(b) .
$$

Then multiplying both hand sides of $(2.5)$ by $t^{\lambda-1} \mathcal{F}_{\rho, \lambda}^{\sigma}\left[\omega(b-a)^{\rho} t^{\rho}\right]$ and integrating the resulting inequality with respect to $t$ over $[0,1]$, we obtain

$$
\begin{aligned}
& \int_{0}^{1} t^{\lambda-1} \mathcal{F}_{\rho, \lambda}^{\sigma}\left[\omega(b-a)^{\rho} t^{\rho}\right] \varphi(t a+(1-t) b) d t \\
& +\int_{0}^{1} t^{\lambda-1} \mathcal{F}_{\rho, \lambda}^{\sigma}\left[\omega(b-a)^{\rho} t^{\rho}\right] \varphi((1-t) a+t b) d t \\
& \leq[\varphi(a)+\varphi(b)] \int_{0}^{1} t^{\lambda-1} \mathcal{F}_{\rho, \lambda}^{\sigma}\left[\omega(b-a)^{\rho} t^{\rho}\right] d t .
\end{aligned}
$$

Using the similar arguments as above we can show that

$$
\frac{1}{(b-a)^{\lambda}}\left[\left(\partial_{\rho, \lambda, a+; \omega}^{\sigma} \varphi\right)(b)+\left(\partial_{\rho, \lambda, b^{-} ; \omega}^{\sigma} \varphi\right)(a)\right] \leq \mathcal{F}_{\rho, \lambda+1}^{\sigma}\left[\omega(b-a)^{\rho}\right][\varphi(a)+\varphi(b)]
$$

and the second inequality is proved.

Remark 2.1. If in Theorem 2.1 we set $\lambda=\alpha, \sigma(0)=1, w=0$, then the inequalities (2.1) become the inequalities (1.4) of Theorem 1.2.

Remark 2.2. If in Theorem 2.1 we set $\lambda=1, \sigma(0)=1, w=0$, then the inequalities (2.1) become the inequalities (1.1).

Before starting and proving our next result, we need the following lemma.

Lemma 2.1. Let $\varphi:[a, b] \rightarrow \mathbb{R}$ be a differentiable mapping on $(a, b)$ with $a<b$ and $\lambda>0$. If $\varphi^{\prime} \in L[a, b]$, then the following equality for fractional integrals holds:

$$
\frac{\varphi(a)+\varphi(b)}{2}-\frac{1}{2(b-a)^{\lambda} \mathcal{F}_{\rho, \lambda+1}^{\sigma}\left[\omega(b-a)^{\rho}\right]}\left[\left(\partial_{\rho, \lambda, a+; \omega}^{\sigma} \varphi\right)(b)+\left(\partial_{\rho, \lambda, b^{-} ; \omega}^{\sigma} \varphi\right)(a)\right]
$$




$$
\begin{aligned}
= & \frac{(b-a)}{2 \mathcal{F}_{\rho, \lambda+1}^{\sigma}\left[\omega(b-a)^{\rho}\right]}\left[\int_{0}^{1}(1-t)^{\lambda} \mathcal{F}_{\rho, \lambda+1}^{\sigma}\left[\omega(b-a)^{\rho}(1-t)^{\rho}\right] \varphi^{\prime}(t a+(1-t) b) d t\right. \\
& \left.-\int_{0}^{1} t^{\lambda} \mathcal{F}_{\rho, \lambda+1}^{\sigma}\left[\omega(b-a)^{\rho} t^{\rho}\right] \varphi^{\prime}(t a+(1-t) b) d t\right] .
\end{aligned}
$$

Proof. Here, we apply integration by parts in integrals of right hand side of (2.6), then we have

$$
\begin{aligned}
& \int_{0}^{1}(1-t)^{\lambda} \mathcal{F}_{\rho, \lambda+1}^{\sigma}\left[\omega(b-a)^{\rho}(1-t)^{\rho}\right] \varphi^{\prime}(t a+(1-t) b) d t \\
& -\int_{0}^{1} t^{\lambda} \mathcal{F}_{\rho, \lambda+1}^{\sigma}\left[\omega(b-a)^{\rho} t^{\rho}\right] \varphi^{\prime}(t a+(1-t) b) d t \\
& =\left.(1-t)^{\lambda} \mathcal{F}_{\rho, \lambda+1}^{\sigma}\left[\omega(b-a)^{\rho}(1-t)^{\rho}\right] \frac{\varphi(t a+(1-t) b)}{a-b}\right|_{0} ^{1} \\
& -\frac{1}{b-a} \int_{0}^{1}(1-t)^{\lambda-1} \mathcal{F}_{\rho, \lambda}^{\sigma}\left[\omega(b-a)^{\rho}(1-t)^{\rho}\right] \varphi(t a+(1-t) b) d t \\
& +\left.t^{\lambda} \mathcal{F}_{\rho, \lambda+1}^{\sigma}\left[\omega(b-a)^{\rho} t^{\rho}\right] \frac{\varphi(t a+(1-t) b)}{b-a}\right|_{0} ^{1} \\
& -\frac{1}{b-a} \int_{0}^{1} t^{\lambda-1} \mathcal{F}_{\rho, \lambda}^{\sigma}\left[\omega(b-a)^{\rho} t^{\rho}\right] \varphi(t a+(1-t) b) d t .
\end{aligned}
$$

Now we use the substitution rule last integrals in (2.7), after by using definition of left and right-sided fractional integral operator, we obtain proof of this lemma.

Remark 2.3. If in Lemma 2.1 we set $\lambda=\alpha, \sigma(0)=1$, and $w=0$, then the inequalities (2.6) become the equality (1.5) of Lemma 1.2.

Remark 2.4. If in Lemma 2.1 we set $\lambda=1, \sigma(0)=1$, and $w=0$, then the inequalities (2.6) become the equality (1.2) of Lemma 1.1.

We have the following results.

Theorem 2.2. Let $\varphi:[a, b] \rightarrow \mathbb{R}$ be a differentiable mapping on $(a, b)$ with $a<b$ and $\lambda>0$. If $\left|\varphi^{\prime}\right|$ is convex on $[a, b]$, then the following inequality for fractional integrals holds:

$$
\left|\frac{\varphi(a)+\varphi(b)}{2}-\frac{1}{2(b-a)^{\lambda} \mathcal{F}_{\rho, \lambda+1}^{\sigma}\left[\omega(b-a)^{\rho}\right]}\left[\left(\partial_{\rho, \lambda, a+; \omega}^{\sigma} \varphi\right)(b)+\left(\partial_{\rho, \lambda, b^{-;}, \omega}^{\sigma} \varphi\right)(a)\right]\right|
$$




$$
\leq(b-a) \frac{\mathcal{F}_{\rho, \lambda+2}^{\sigma^{\prime}}\left[\omega(b-a)^{\rho}\right]}{\mathcal{F}_{\rho, \lambda+1}^{\sigma}\left[\omega(b-a)^{\rho}\right]} \frac{\left|\varphi^{\prime}(a)\right|+\left|\varphi^{\prime}(b)\right|}{2},
$$

where

$$
\sigma^{\prime}(k):=\sigma(k)\left(1-\frac{1}{2^{\rho k+\lambda}}\right) .
$$

Proof. Using Lemma 2.1 and the convexity of $\left|\varphi^{\prime}\right|$, we find that

$$
\begin{aligned}
& \left|\frac{\varphi(a)+\varphi(b)}{2}-\frac{1}{2(b-a)^{\lambda} \mathcal{F}_{\rho, \lambda+1}^{\sigma}\left[\omega(b-a)^{\rho}\right]}\left[\left(\mathcal{J}_{\rho, \lambda, a+; \omega}^{\sigma} \varphi\right)(b)+\left(\mathcal{J}_{\rho, \lambda, b^{-} ; \omega}^{\sigma} \varphi\right)(a)\right]\right| \\
\leq & \frac{(b-a)}{2 \mathcal{F}_{\rho, \lambda+1}^{\sigma}\left[\omega(b-a)^{\rho}\right]}\left[\sum_{k=0}^{\infty} \frac{\sigma(k) \omega^{k}(b-a)^{\rho k}}{\Gamma(\rho k+\lambda+1)}\right. \\
& \left.\left.\times \int_{0}^{1}\left|(1-t)^{\rho k+\lambda}-t^{\rho k+\lambda}\right|\left[t\left|\varphi^{\prime}(a)\right|+(1-t)\left|\varphi^{\prime}(b)\right|\right] d t\right)\right] \\
= & \frac{(b-a)}{2 \mathcal{F}_{\rho, \lambda+1}^{\sigma}\left[\omega(b-a)^{\rho}\right]}\left[\sum_{k=0}^{\infty} \frac{\sigma(k) \omega^{k}(b-a)^{\rho k}}{\Gamma(\rho k+\lambda+1)}\right. \\
& \times\left\{\int_{0}^{\frac{1}{2}}\left[(1-t)^{\rho k+\lambda}-t^{\rho k+\lambda}\right]\left[t\left|\varphi^{\prime}(a)\right|+(1-t)\left|\varphi^{\prime}(b)\right|\right] d t\right. \\
& \left.\left.+\int_{\frac{1}{2}}^{1}\left[t^{\rho k+\lambda}-(1-t)^{\rho k+\lambda}\right]\left[t\left|\varphi^{\prime}(a)\right|+(1-t)\left|\varphi^{\prime}(b)\right|\right] d t\right\}\right] \\
= & \frac{(b-a)}{2 \mathcal{F}_{\rho, \lambda+1}^{\sigma}\left[\omega(b-a)^{\rho}\right]}\left(\mathcal{F}_{\rho, \lambda+2}^{\sigma^{\prime}}\left[\omega(b-a)^{\rho}\right]\right)\left(\left|\varphi^{\prime}(a)\right|+\left|\varphi^{\prime}(b)\right|\right) .
\end{aligned}
$$

This completes the proof.

Remark 2.5. If in Theorem 2.2 we set $\lambda=\alpha, \sigma(0)=1$, and $w=0$, then the inequality (2.8) become the inequalities (1.6) of Theorem 1.3.

Remark 2.6. If in Theorem 2.2 we set $\lambda=1, \sigma(0)=1$, and $w=0$, then, the inequality (2.8) become the inequalities (1.3) of Theorem 1.1.

Theorem 2.3. Let $\varphi:[a, b] \rightarrow \mathbb{R}$ be a differentiable mapping on $(a, b)$ with $a<b$. If $\left|\varphi^{\prime}\right|^{q}$ is convex on $[a, b]$ for some $q>1$, then the following inequality for fractional integrals holds:

$$
\begin{aligned}
& \left|\frac{\varphi(a)+\varphi(b)}{2}-\frac{1}{2(b-a)^{\lambda} \mathcal{F}_{\rho, \lambda+1}^{\sigma}\left[w(b-a)^{\rho}\right]}\left[\left(\mathcal{J}_{\rho, \lambda, a+; w}^{\sigma} \varphi\right)(b)+\left(\mathcal{J}_{\rho, \lambda, b^{-} ; w}^{\sigma} \varphi\right)(a)\right]\right| \\
\leq & \frac{(b-a)}{2 \mathcal{F}_{\rho, \lambda+1}^{\sigma}\left[w(b-a)^{\rho}\right]} \mathcal{F}_{\rho, \lambda+1}^{\sigma_{1}}\left[w(b-a)^{\rho}\right]
\end{aligned}
$$




$$
\times\left[\left(\frac{\left|\varphi^{\prime}(a)\right|^{q}+3\left|\varphi^{\prime}(b)\right|^{q}}{8}\right)^{\frac{1}{q}}+\left(\frac{3\left|\varphi^{\prime}(a)\right|^{q}+\left|\varphi^{\prime}(b)\right|^{q}}{8}\right)^{\frac{1}{q}}\right],
$$

where

$$
\sigma_{1}(k):=\sigma(k)\left(\frac{1}{(\rho k+\lambda) p+1}\right)^{\frac{1}{p}}\left(1-\frac{1}{2^{(\rho k+\lambda) p}}\right)^{\frac{1}{p}},
$$

with $\frac{1}{p}+\frac{1}{q}=1, \lambda>0$.

Proof. Using Lemma 2.1 and the convexity of $\left|\varphi^{\prime}\right|^{q}$, and Hölder's inequality, we obtain

$$
\begin{aligned}
& \left|\frac{\varphi(a)+\varphi(b)}{2}-\frac{1}{2(b-a)^{\lambda} \mathcal{F}_{\rho, \lambda+1}^{\sigma}\left[\omega(b-a)^{\rho}\right]}\left[\left(\partial_{\rho, \lambda, a+; \omega}^{\sigma} \varphi\right)(b)+\left(\partial_{\rho, \lambda, b^{-} ; \omega}^{\sigma} \varphi\right)(a)\right]\right| \\
& \leq \frac{(b-a)}{2 \mathcal{F}_{\rho, \lambda+1}^{\sigma}\left[\omega(b-a)^{\rho}\right]}\left[\sum_{k=0}^{\infty} \frac{\sigma(k) \omega^{k}(b-a)^{\rho k}}{\Gamma(\rho k+\lambda+1)}\right. \\
& \times\left\{\left(\int_{0}^{\frac{1}{2}}\left[(1-t)^{\rho k+\lambda}-t^{\rho k+\lambda}\right]^{p} d t\right)^{\frac{1}{p}}\left(\int_{0}^{\frac{1}{2}}\left[t\left|\varphi^{\prime}(a)\right|^{q}+(1-t)\left|\varphi^{\prime}(b)\right|^{q}\right] d t\right)^{\frac{1}{q}}\right. \\
& \left.\left.+\left(\int_{\frac{1}{2}}^{1}\left[t^{\rho k+\lambda}-(1-t)^{\rho k+\lambda}\right]^{p} d t\right)^{\frac{1}{p}}\left(\int_{\frac{1}{2}}^{1}\left[t\left|\varphi^{\prime}(a)\right|^{q}+(1-t)\left|\varphi^{\prime}(b)\right|^{q}\right] d t\right)^{\frac{1}{q}}\right\}\right] \\
& \leq \frac{(b-a)}{2 \mathcal{F}_{\rho, \lambda+1}^{\sigma}\left[\omega(b-a)^{\rho}\right]}\left[\sum_{k=0}^{\infty} \frac{\sigma(k) \omega^{k}(b-a)^{\rho k}}{\Gamma(\rho k+\lambda+1)}\right. \\
& \times\left\{\left(\int_{0}^{\frac{1}{2}}\left[(1-t)^{(\rho k+\lambda) p}-t^{(\rho k+\lambda) p}\right] d t\right)^{\frac{1}{p}}\left(\int_{0}^{\frac{1}{2}}\left[t\left|\varphi^{\prime}(a)\right|^{q}+(1-t)\left|\varphi^{\prime}(b)\right|^{q}\right] d t\right)^{\frac{1}{q}}\right. \\
& \left.\left.+\left(\int_{\frac{1}{2}}^{1}\left[t^{(\rho k+\lambda) p}-(1-t)^{(\rho k+\lambda) p}\right] d t\right)^{\frac{1}{p}}\left(\int_{\frac{1}{2}}^{1}\left[t\left|\varphi^{\prime}(a)\right|^{q}+(1-t)\left|\varphi^{\prime}(b)\right|^{q}\right] d t\right)^{\frac{1}{q}}\right\}\right] \\
& =\frac{(b-a)}{2 \mathcal{F}_{\rho, \lambda+1}^{\sigma}\left[w(b-a)^{\rho}\right]} \mathcal{F}_{\rho, \lambda+1}^{\sigma_{1}}\left[w(b-a)^{\rho}\right] \\
& \times\left[\left(\frac{\left|\varphi^{\prime}(a)\right|^{q}+3\left|\varphi^{\prime}(b)\right|^{q}}{8}\right)^{\frac{1}{q}}+\left(\frac{3\left|\varphi^{\prime}(a)\right|^{q}+\left|\varphi^{\prime}(b)\right|^{q}}{8}\right)^{\frac{1}{q}}\right] .
\end{aligned}
$$

Here, we use $(A-B)^{p} \leq A^{p}-B^{p}$ for any $A>B \geq 0$ and $p \geq 1$.

This completes the proof. 
Corollary 2.1. Under the assumption of Theorem 2.3 with $\lambda=\alpha, \sigma(0)=1$ and $w=0$, we have

$$
\begin{aligned}
& \left|\frac{\varphi(a)+\varphi(b)}{2}-\frac{\Gamma(\alpha+1)}{2(b-a)^{\alpha}}\left[J_{a+}^{\alpha} \varphi(b)+J_{b-}^{\alpha} \varphi(a)\right]\right| \\
\leq & \frac{(b-a)}{2}\left(\frac{1}{\alpha p+1}\right)^{\frac{1}{p}}\left(1-\frac{1}{2^{\alpha p}}\right)^{\frac{1}{p}} \\
& \times\left[\left(\frac{\left|\varphi^{\prime}(a)\right|^{q}+3\left|\varphi^{\prime}(b)\right|^{q}}{8}\right)^{\frac{1}{q}}+\left(\frac{3\left|\varphi^{\prime}(a)\right|^{q}+\left|\varphi^{\prime}(b)\right|^{q}}{8}\right)^{\frac{1}{q}}\right] .
\end{aligned}
$$

Corollary 2.2. If we take $\alpha=1$ in Corollary 2.1, we have

$$
\begin{aligned}
& \left|\frac{\varphi(a)+\varphi(b)}{2}-\frac{1}{(b-a)} \int_{a}^{b} \varphi(t) d t\right| \\
\leq & \left(\frac{b-a}{2}\right)\left(\frac{1}{p+1}\right)^{\frac{1}{p}}\left(1-\frac{1}{2^{p}}\right)^{\frac{1}{p}} \\
& \times\left[\left(\frac{\left|\varphi^{\prime}(a)\right|^{q}+3\left|\varphi^{\prime}(b)\right|^{q}}{8}\right)^{\frac{1}{q}}+\left(\frac{3\left|\varphi^{\prime}(a)\right|^{q}+\left|\varphi^{\prime}(b)\right|^{q}}{8}\right)^{\frac{1}{q}}\right] .
\end{aligned}
$$

\section{REFERENCES}

[1] R. P. Agarwal, M.-J. Luo and R. K. Raina, On Ostrowski type inequalities, Fasc. Math. 56(1) (2016) DOI 10.1515/fascmath-2016-0001.

[2] S. S. Dragomir and R. P. Agarwal, Two inequalities for differentiable mappings and applications to special means of real numbers and to trapezoidal formula, Appl. Math. Lett. 11(5) (1998), 91-95.

[3] A. A. Kilbas, H. M. Srivastava and J. J. Trujillo, Theory and Applications of Fractional Differential Equations, North-Holland Mathematics Studies 204, Elsevier, Amsterdam, 2006.

[4] R. Gorenflo and F. Mainardi, Fractional Calculus: Integral and Differential Equations of Fractional Order, Springer Verlag, Wien, 1997, 223-276.

[5] J. Hadamard, Etude sur les proprietes des fonctions entieres et en particulier d'une fonction considree par, Jornal de Mathematiqués Pures et Apploquées 58 (1893), 171-215.

[6] S. Miller and B. Ross, An Introduction to the Fractional Calculus and Fractional Differential Equations, John Wiley \& Sons, USA, New York, 1993.

[7] R. K. Raina, On generalized Wright's hypergeometric functions and fractional calculus operators, East Asian Mathematical Journal 21(2) (2005), 191-203.

[8] M. Z. Sarikaya, E. Set, H. Yaldiz and N. Basak, Hermite-Hadamard's inequalities for fractional integrals and related fractional inequalities, Math. Comput. Modelling 57 (2013), 2403-2407.

[9] M. Z. Sarikaya and H. Yaldiz, On generalization integral inequalities for fractional integrals, Nihonkai Math. J. 25 (2014), 93-104.

[10] M. Z. Sarikaya, H. Yaldiz and N. Basak, New fractional inequalities of Ostrowski-Grüss type, Le Matematiche 69(1) (2014), 227-235 .

[11] M. Z. Sarikaya and H. Yaldiz, On Hermite-Hadamard type inequalities for $\varphi$-convex functions via fractional integrals, Malays. J. Math. Sci. 9(2) (2015), 243-258. 
${ }^{1}$ Department of Mathematics, University of KARAMANOĞLU Mehmetbey, KARAMANOĞLU Mehmetbey UNIVERSITY,

KAMIL ÖZDAĞ SCIENCE FACULTY,

Department of MATHEMATiCs,

Yunus EMre CAMPUS, 70100 KARAMAN-TURKEY

Email address: yaldizhatice@gmail.com

${ }^{2}$ Department of Mathematics, UNIVERSITY OF DUZCE,

DuZCE UNIVERSITY,

FACUlTy OF SCIENCE AND ARTS, DePARTMENT OF MATHEMATiCs, DUZCE-TURKEY

Email address: sarikayamz@gmail.com 